International Mathematical Forum, 2, 2007, no. 9, 429 - 440

\title{
Ranking Bank Branches with Interval Data The Application of DEA
}

\author{
F. Hosseinzadeh Lotfi $^{1}$ \\ Department of Mathematics, Science and Research Branch, \\ Islamic Azad University, Tehran, Iran \\ M. Navabakhs \\ Department of Society, Science and Research Branch, \\ Islamic Azad University, Tehran, Iran \\ A. Tehranian \\ Department of Mathematics, Science and Research Branch, \\ Islamic Azad University, Tehran, Iran \\ M. Rostamy-Malkhalifeh \\ Department of Mathematics, Science and Research Branch, \\ Islamic Azad University, Tehran, Iran

\section{R. Shahverdi} \\ Department of Mathematics, Science and Research Branch, \\ Islamic Azad University, Tehran, Iran
}

\begin{abstract}
DEA (Data Envelopment Analysis) evaluates the relative efficiency of a set of DMUs (Decision Making Units). The relative efficiency of a DMU is the result of comparing the inputs and outputs of the DMU and those of other DMUs in the PPS (Production Possibility Set). If the inputs and outputs each vary in intervals, the DMUs cannot be easily evaluated and ranked using the obtained efficiency scores. In this paper, presenting a new idea for computing the efficiency of DMUs with
\end{abstract}

\footnotetext{
${ }^{1}$ Corresponding author:Farhad Hosseinzadeh Lotfi, E-mail:hosseinzadeh_lotfi@yahoo.com
} 
interval data, an interval will be defined for the efficiency score of each unit. And finally, a method for ranking DMUs by the obtained efficiency interval is presented. And, the new technique will be applied to a set of real data.

Keywords: Data Envelopment Analysis; Interval data; Ranking

\section{Introduction}

Data Envelopment Analysis technique was presented in the CCR paper by Charnes et al. (1997), and since then was developed by various researchers. In this method, the relative efficiency of a set of DMUs which use similar types of (multiple) resources to produce similar types of (multiple) outputs is computed. Finally, DMUs are divided into two groups of efficient and inefficient DMUs. In ordinary DEA models, the input and output values are assumed to be definite. In recent year, in different applications of DEA, inputs and outputs have been observed whose values are indefinite. Such data are called "inaccurate". Inaccurate data can be probabilistic, interval, ordinal, qualitative, or fuzzy. Therefore, some papers were presented on the theoretical development of this technique whit interval data, of which we can name Despotis et. al(2002)and Jahanshahloo et al. (2004). In the above-mentioned papers, all the DMUs are divided into three groups which are defined according to the interval obtained for the efficiency value of DMUs. This paper consists of the following sections: In section 2, DEA is discussed. DEA with interval data is presented in section3. And, the method for ranking DMUs with interval data is put forward in section4. Finally, an example with real interval data will be given.

\section{DEA}

Suppose that we have $\mathrm{n} \mathrm{DMU}_{\mathrm{s}}, \mathrm{DMU}_{j}: j=1, \ldots, n$, to be evaluated, each DMU using $m$ inputs to produce $s$ outputs. $X_{j}=\left(x_{1 j}, \ldots, x_{m j}\right)$ and $Y_{j}=$ $\left(y_{1 j}, \ldots, y_{s j}\right)$ are the input and output vectors of $\mathrm{DMU}_{j}$, respectively, in which $X_{j}, Y_{j} \geq 0, X_{j} \neq 0$ and $Y_{j} \neq 0$. The input-oriented CCR model to evaluate the relative efficiency of DMUp is as follows: 


$$
\begin{aligned}
e_{p}=\max & \sum_{r=1}^{s} u_{r} y_{r p} \\
\text { s.t. } & \sum_{r=1}^{s} u_{r} y_{r j}-\sum_{i=1}^{m} v_{i} x_{i j} \leq 0, \quad j=1, \ldots, n, \\
& \sum_{i=1}^{m} v_{i} x_{i p}=1 \\
& u_{i}, v_{r} \geq \varepsilon
\end{aligned}
$$

where $\mathrm{U}=\left(u_{1}, \ldots, u_{s}\right)$ and $V=\left(v_{1}, \ldots, v_{m}\right)$ are output and input weight vectors, respectively, and they are unknown, and should be computed by solving problem (1). Assume that $\left(U^{*}, V^{*}\right)$ is the optimal solution to problem (1). Then, the $D M U_{p}$ under evaluation is relative efficient if and only if $e_{p}^{*}=1$.Otherwise, DMU is said to be inefficient. In problem (1), it is assumed that all inputs and outputs of any DMU are of known values.

\section{Interval DEA}

Let input and output values of any DMU be located in a certain interval, where $x_{i j}^{L}$ and $x_{i j}^{U}$ are the lower and upper bounds of the $i$ th input of the $j$ th DMU, respectively, and $y_{r j}^{L}$ and $y_{r j}^{U}$ are the lower and upper bounds of the $r$ th output of the $j$ th DMU, respectively; that is to say, $x_{i j}^{L} \leq x_{i j} \leq x_{i j}^{U}$ and $y_{i j}^{L} \leq y_{i j} \leq y_{i j}^{U}$. Such data are called interval data, because they are located in intervals. Note that always $x_{i j}^{L} \leq x_{i j}^{U}$ and $y_{i j}^{L} \leq y_{i j}^{U}$. If $x_{i j}^{L}=x_{i j}^{U}$, then the $i$ th input of the $j$ th DMU has a definite value. Interval problems are those whose parameter values are located in intervals, their exact values being unable to be identified. The CCR model for evaluating $D M U_{p}$ with interval data is as follows:

$$
\begin{aligned}
e_{p}=\max & \sum_{r=1}^{s} u_{r}\left[y_{r p}^{L}, y_{r p}^{U}\right] \\
\text { s.t. } & \sum_{\substack{s=1 \\
r=1}}^{m} u_{r}\left[y_{r j}^{L}, y_{r j}^{U}\right]-\sum_{i=1}^{m} v_{i}\left[x_{i j}^{L}, x_{i j}^{U}\right] \leq 0, \quad j=1, \ldots, n, \\
& \sum_{i=1}^{m} v_{i}\left[x_{i p}^{L}, x_{i p}^{U}\right]=1 \\
& u_{i}, v_{r} \geq \varepsilon
\end{aligned}
$$


In problem (2), seeing that all parameters of the problem are in intervals and do not have definite values, the relative efficiency of $D M U_{p}$ is also located in an interval. The upper and lower bounds of the relative efficiency of $D M U_{p}$ are obtained by solving the following problems, respectively.

$$
\begin{aligned}
e_{p}^{U}=\max & \sum_{r=1}^{s} u_{r} y_{r p}^{U} \\
\text { s.t. } & \sum_{r=1}^{s} u_{r} y_{r j}^{L}-\sum_{i=1}^{m} v_{i} x_{i j}^{U} \leq 0, \quad j=1, \ldots, n, j \neq p \\
& \sum_{r=1}^{s} u_{r} y_{r p}^{U}-\sum_{i=1}^{m} v_{i} x_{i p}^{L} \leq 0, \\
& \sum_{i=1}^{m} v_{i} x_{i p}^{L}=1 \\
& u_{i}, v_{r} \geq \varepsilon \\
e_{p}^{L}=\max & \sum_{r=1}^{s} u_{r} y_{r p}^{L} \\
\text { s.t. } & \sum_{r=1}^{s} u_{r} y_{r j}^{U}-\sum_{i=1}^{m} v_{i} x_{i j}^{L} \leq 0, \quad j=1, \ldots, m, \quad r=1, \ldots, s, j \neq p \\
& \sum_{r=1}^{s} u_{r} y_{r p}^{L}-\sum_{i=1}^{m} v_{i} x_{i p}^{U} \leq 0, \\
& \sum_{i=1}^{m} v_{i} x_{i p}^{U}=1 \\
& u_{i}, v_{r} \geq \varepsilon \\
&
\end{aligned}
$$

In problem (3), the DMU under evaluation is in its best condition, and the other DMUs are in their worst condition. So, $e_{p} \leq e_{p}^{U}$ always holds. Whereas, in problem (4), the DMU under evaluation is in its worst condition and the other DMUs are in their best condition. Therefore, the obtained efficiency will be the worst possible efficiency for the DMU under evaluation, so $e_{p} \geq e_{p}^{L}$. According to what has been mentioned, we can say, $e_{p} \in\left[e_{p}^{L}, e_{p}^{U}\right]$. Considering that the efficiency of any DMU lies in an interval, all DMUs can be divided into one of the three following classes:

Class 1 includes all DMUs which are efficient both in their best and worst 
conditions, that is to say, $E^{++}=\left\{D M U j: e_{j}^{L}=e_{j}^{U}=1\right\}$.

Class 2 consists of all DMUs which are efficient in their best condition, but inefficient in their worst condition, in other words, $E^{+}=\left\{D M U j: e_{j}^{L}<1, e_{j}^{U}=\right.$ $1\}$.

And, class 3 contains all DMUs which are inefficient in their best condition. It goes without saying that such DMUs are, also, inefficient in their best condition, that is to say, $E^{-}=\left\{D M U j: e_{j}^{L}<1, e_{j}^{U}<1\right\}$. All DMUs in $E^{++}$are as efficient DMUs, since they are efficient both in their best and worst conditions. And, all DMUs in $E^{-}$are inefficient, as they are inefficient both in their best and worst conditions. But, in case of DMUs in $E^{+}$, one cannot determine their being efficient or inefficient, because they are efficient in some condition and inefficient in some other condition. In the next section, a method will be presented for ranking DMUs with interval data.

\section{Ranking DMUs with interval data}

In the previous section, a method was presented by which interval efficiency was obtained for every DMU with interval data. Ranking interval efficiency seems a little difficult. In other words, if two DMUs are located in $E^{++}$, how can we comment on one of them being better than the other? The same holds true for DMUs in $E^{+}$or in $E^{-}$. In this section, a method is proposed for ranking the DMUs in each class. To being with, suppose that any DMU in $E^{+}$has a better rank than DMUs in $E^{-}$. Therefore, the following proposed method can be applied to each one of the classes $E^{++}, E^{+}$and $E^{-}$, separately. For each DMU, four types of efficiency can be computed:

a) the DMUs under evaluation in its best condition, and the other DMUs in their worst condition,

$$
\begin{aligned}
& e_{p}^{1}=\max \sum_{r=1}^{s} u_{r} y_{r p}^{U} \\
& \text { s.t. } \sum_{r=1}^{s} u_{r} y_{r j}^{L}-\sum_{i=1}^{m} v_{i} x_{i j}^{U} \leq 0, \quad j=1, \ldots, n, j \neq p \\
& \sum_{r=1}^{s} u_{r} y_{r p}^{U}-\sum_{i=1}^{m} v_{i} x_{i p}^{L} \leq 0 \\
& \sum_{i=1}^{m} v_{i} x_{i p}^{L}=1 \\
& u_{i}, v_{r} \geq \varepsilon
\end{aligned}
$$


b) the DMU under evaluation in its best condition, and the other DMUs, also, in their best condition,

$$
\begin{aligned}
& e_{p}^{2}=\max \sum_{r=1}^{s} u_{r} y_{r p}^{U} \\
& \text { s.t. } \sum_{r=1}^{s} u_{r} y_{r j}^{U}-\sum_{i=1}^{m} v_{i} x_{i j}^{L} \leq 0, \quad j=1, \ldots, n \text {, } \\
& \sum_{i=1}^{m} v_{i} x_{i p}^{L}=1 \\
& u_{i}, v_{r} \geq \varepsilon \quad i=1, \ldots, m, r=1, \ldots, s,
\end{aligned}
$$

c) the DMU under evaluation in its worst condition, and the other DMUs, also, in their worst condition,

$$
\begin{aligned}
& e_{p}^{3}=\max \sum_{r=1}^{s} u_{r} y_{r p}^{L} \\
& \text { s.t. } \sum_{\substack{r=1 \\
m}}^{s} u_{r} y_{r j}^{L}-\sum_{i=1}^{m} v_{i} x_{i j}^{U} \leq 0, \quad j=1, \ldots, n \text {, } \\
& \sum_{i=1}^{m} v_{i} x_{i p}^{U}=1 \\
& u_{i}, v_{r} \geq \varepsilon \quad i=1, \ldots, m, r=1, \ldots, s,
\end{aligned}
$$

d) the DMU under evaluation in its worst condition, and the other DMUs in their best condition,

$$
\begin{aligned}
e_{p}^{4}=\max & \sum_{r=1}^{s} u_{r} y_{r p}^{L} \\
\text { s.t. } & \sum_{r=1}^{s} u_{r} y_{r j}^{U}-\sum_{i=1}^{m} v_{i} x_{i j}^{L} \leq 0, \quad j=1, \ldots, n, j \neq p \\
& \sum_{r=1}^{s=1} u_{r} y_{r p}^{L}-\sum_{i=1}^{m} v_{i} x_{i p}^{U} \leq 0, \\
& \sum_{i=1}^{m} v_{i} x_{i p}^{U}=1 \\
& u_{i}, v_{r} \geq \varepsilon
\end{aligned}
$$


Considering problems (5), (6), (7) and (8), we can state the following: $e_{p}^{1}=e_{p}^{U}, e_{p}^{4}=e_{p}^{L}, e_{p}^{4} \leq e_{p}^{2} \leq e_{p}^{1}, e_{p}^{4} \leq e_{p}^{3}, e_{p}^{3} \leq e_{p}^{1}$. The following method is proposed for ranking DMUs in each class $E^{++}, E^{+}$and $E^{-}$. First, solve the four following models which correspond each DMU, respectively. The models presented below are the AP models corresponding problems (5), (6), (7) and (8).

$$
\begin{aligned}
& \theta_{p}^{1}=\max \sum_{r=1}^{s} u_{r} y_{r p}^{U} \\
& \text { s.t. } \sum_{r=1}^{s} u_{r} y_{r j}^{L}-\sum_{i=1}^{m} v_{i} x_{i j}^{U} \leq 0, \quad j=1, \ldots, n, j \neq p \\
& \sum_{i=1}^{m} v_{i} x_{i p}^{L}=1 \\
& u_{i}, v_{r} \geq \varepsilon \quad i=1, \ldots, m, r=1, \ldots, s,
\end{aligned}
$$

$$
\begin{aligned}
\theta_{p}^{2}=\max & \sum_{r=1}^{s} u_{r} y_{r p}^{U} \\
\text { s.t. } & \sum_{r=1}^{s=1} u_{r} y_{r j}^{U}-\sum_{i=1}^{m} v_{i} x_{i j}^{L} \leq 0, \quad j=1, \ldots, n, j \neq p \\
& \sum_{i=1}^{m} v_{i} x_{i p}^{L}=1 \\
& \\
u_{i}, v_{r} \geq \varepsilon & i=1, \ldots, m, \quad r=1, \ldots, s,
\end{aligned}
$$




$$
\begin{aligned}
& \theta_{p}^{3}=\max \sum_{r=1}^{s} u_{r} y_{r p}^{L} \\
& \text { s.t. } \sum_{r=1}^{s} u_{r} y_{r j}^{L}-\sum_{i=1}^{m} v_{i} x_{i j}^{U} \leq 0, \quad j=1, \ldots, n, j \neq p \\
& \sum_{i=1}^{m} v_{i} x_{i p}^{U}=1 \\
& u_{i}, v_{r} \geq \varepsilon \quad i=1, \ldots, m, r=1, \ldots, s, \\
& \theta_{p}^{4}=\max \sum_{r=1}^{s} u_{r} y_{r p}^{L} \\
& \text { s.t. } \sum_{r=1}^{s} u_{r} y_{r j}^{U}-\sum_{i=1}^{m} v_{i} x_{i j}^{L} \leq 0, \quad j=1, \ldots, n, j \neq p \\
& \sum_{i=1}^{m} v_{i} x_{i p}^{U}=1 \\
& u_{i}, v_{r} \geq \varepsilon \quad i=1, \ldots, m, r=1, \ldots, s,
\end{aligned}
$$

Problems (9), (10), (11) and (12) are the very problems (5), (6), (7) and (8), except that the boundedness condition of the objective function has been excluded from the constraints. Therefore, the values of $\theta_{p}^{1}, \theta_{p}^{2}, \theta_{p}^{3}$ and $\theta_{p}^{4}$ may exceed unity. Now the following criterion can be suggested for ranking the DMUs of each class.

$$
\theta_{p}=\frac{1}{\sum_{i=1}^{4} \alpha_{i}}\left(\alpha_{1} \theta_{p}^{1}+\alpha_{2} \theta_{p}^{2}+\alpha_{3} \theta_{p}^{3}+\alpha_{4} \theta_{p}^{4}\right) \text {. }
$$

In a certain class, any DMU having a higher value will have a better ranking. The application of the above method for ranking should be carried out separately to each class, and ranking should be done separately in each class.

\section{$5 \quad$ Ranking Bank Branches}

We now apply our approach to some commercial bank branches in Iran. There are 20 branches in this district. Each branch uses 3 inputs to produce 5 
outputs. Table 1 shows these inputs and outputs.

\begin{tabular}{|l|l|}
\hline Inputs & Outputs \\
\hline Payable interest & The total sum of four main deposits \\
Personnel & Other deposits \\
Non-Performing loans & Loans granted \\
& Received interest \\
& Fee \\
\hline
\end{tabular}

Table 1 Inputs and Outputs

In Tables 2 and 3 the interval inputs and interval outputs for these DMUs are given. Also in Table 4 the efficiencies of these DMUs are presented.

\begin{tabular}{|c|c|c|c|c|c|c|}
\hline$D M U_{j}$ & $x_{1 j}^{L}$ & $x_{2 j}^{U}$ & $x_{2 j}^{L}$ & $x_{2 j}^{U}$ & $x_{3 j}^{L}$ & $x_{3 j}^{U}$ \\
\hline 1 & 5007.37 & 9613.37 & 36.29 & 36.86 & 87243 & 87243 \\
2 & 2926.81 & 5961.55 & 18.8 & 2016 & 9945 & 12120 \\
3 & 8732.7 & 17752.5 & 25.74 & 27.17 & 47575 & 50013 \\
4 & 945.93 & 1966.39 & 20.81 & 22.54 & 19292 & 19753 \\
5 & 8487.07 & 17521.66 & 14.16 & 14.8 & 3428 & 3911 \\
6 & 13759.35 & 27359.36 & 19.46 & 19.46 & 13929 & 15657 \\
7 & 587.69 & 1205.47 & 27.29 & 27.48 & 27827 & 29005 \\
8 & 4646.39 & 9559.61 & 24.52 & 25.07 & 9070 & 9983 \\
9 & 1554.29 & 3427.89 & 20.47 & 21.59 & 412036 & 413902 \\
10 & 17528.31 & 36297.54 & 14.84 & 15.05 & 8638 & 10229 \\
11 & 2444.34 & 4955.78 & 20.42 & 20.54 & 500 & 937 \\
12 & 7303.27 & 14178.11 & 22.87 & 23.19 & 16148 & 21353 \\
13 & 9852.15 & 19742.89 & 18.47 & 21.83 & 17163 & 17290 \\
14 & 4540.75 & 9312.24 & 22.83 & 23.96 & 17918 & 17964 \\
15 & 3039.58 & 6304.01 & 39.32 & 39.86 & 51582 & 55136 \\
16 & 6585.81 & 13453.58 & 25.57 & 26.52 & 20975 & 23992 \\
17 & 4209.18 & 8603.79 & 27.59 & 27.95 & 41960 & 43103 \\
18 & 1015.52 & 2037.82 & 13.63 & 13.93 & 18641 & 19354 \\
19 & 5800.38 & 11875.39 & 27.12 & 27.26 & 19500 & 19569 \\
20 & 1445.68 & 2922.15 & 28.96 & 28.96 & 31700 & 32061 \\
\hline
\end{tabular}

Table 2 Input - data for the 20 bank branches 


\begin{tabular}{|c|c|c|c|c|c|c|c|c|c|c|}
\hline$D M U_{j}$ & $y_{1 j}^{L}$ & $y_{1 j}^{U}$ & $y_{2 j}^{L}$ & $y_{2 j}^{U}$ & $y_{3 j}^{L}$ & $y_{3 j}^{U}$ & $y_{4 j}^{L}$ & $y_{4 j}^{U}$ & $y_{5 j}^{L}$ & $y_{5 j}^{U}$ \\
\hline 1 & 2696995 & 3126798 & 263643 & 382545 & 1675519 & 1853365 & 108634.76 & 125740.28 & 965.97 & 6957.33 \\
2 & 340377 & 440355 & 95978 & 117659 & 377309 & 390203 & 32396.65 & 37836.56 & 304.67 & 749.4 \\
3 & 1027546 & 1061260 & 37911 & 503089 & 1233548 & 1822028 & 96842.33 & 108080.01 & 2285.03 & 3174 \\
4 & 1145235 & 1213541 & 229646 & 268460 & 468520 & 542101 & 32362.8 & 39273.37 & 207.98 & 510.93 \\
5 & 390902 & 395241 & 4924 & 12136 & 129751 & 142873 & 12662.71 & 14165.44 & 63.32 & 92.3 \\
6 & 988115 & 1087392 & 74133 & 111324 & 507502 & 574355 & 53591.3 & 72257.28 & 480.16 & 869.52 \\
7 & 144906 & 165818 & 180530 & 180617 & 288513 & 323721 & 40507.97 & 45847.48 & 176.58 & 370.81 \\
8 & 408163 & 416416 & 405396 & 486431 & 1044221 & 1071812 & 56260.09 & 73948.09 & 4654.71 & 5882.53 \\
9 & 335070 & 410427 & 337971 & 449336 & 1584722 & 1802942 & 176436.81 & 189006.12 & 560.26 & 2506.67 \\
10 & 700842 & 768593 & 14378 & 15192 & 2290745 & 2573512 & 662725.21 & 791463.08 & 58.89 & 86.86 \\
11 & 641680 & 696338 & 114183 & 241081 & 1579961 & 2285079 & 17527.58 & 20773.91 & 1070.81 & 2283.08 \\
12 & 453170 & 481943 & 27196 & 29553 & 245726 & 275717 & 35757.83 & 42790.14 & 375.07 & 559.85 \\
13 & 553167 & 574989 & 21298 & 23043 & 425886 & 431815 & 45652.24 & 50255.75 & 438.43 & 836.82 \\
14 & 309670 & 342598 & 20168 & 26172 & 124188 & 126930 & 8143.79 & 11948.04 & 936.62 & 1468.45 \\
15 & 286149 & 317186 & 149183 & 270708 & 787959 & 810088 & 106798.63 & 111962.3 & 1203.79 & 4335.24 \\
16 & 321435 & 347848 & 66169 & 80453 & 360880 & 379488 & 89971.47 & 165524.22 & 200.36 & 399.8 \\
17 & 618105 & 835839 & 244250 & 404579 & 9136507 & 9136507 & 33036.79 & 41826.51 & 2781.24 & 4555.42 \\
18 & 248125 & 320974 & 3063 & 6330 & 26687 & 29173 & 9525.6 & 10877.78 & 240.04 & 274.7 \\
19 & 640890 & 679916 & 490508 & 684372 & 2946797 & 3985900 & 66097.16 & 95329.87 & 961.56 & 1914.25 \\
20 & 119948 & 120208 & 14943 & 17495 & 297674 & 308012 & 21991.53 & 27934.19 & 282.73 & 471.22 \\
\hline
\end{tabular}

Table 3 Output - data for the 20 bank branches

\begin{tabular}{|c|c|c|c|c|}
\hline$D M U_{j}$ & $e_{j}^{1}$ & $e_{j}^{2}$ & $e_{j}^{3}$ & $e_{j}^{4}$ \\
\hline 1 & 1 & 1 & 1 & 1 \\
2 & 1 & 0.657024 & 0.455349 & 0.371424 \\
3 & 1 & 1 & 0.83911 & 0.523443 \\
4 & 1 & 1 & 1 & 1 \\
5 & 0.763021 & 0.666492 & 0.71051 & 0.618831 \\
6 & 1 & 1 & 1 & 0.917738 \\
7 & 1 & 1 & 1 & 0.728914 \\
8 & 1 & 1 & 1 & 1 \\
9 & 1 & 1 & 1 & 1 \\
10 & 1 & 1 & 1 & 1 \\
11 & 1 & 1 & 1 & 1 \\
12 & 0.496069 & 0.396102 & 0.403706 & 0.328345 \\
13 & 0.701987 & 0.53513 & 0.538834 & 0.449969 \\
14 & 0.724067 & 0.353113 & 0.368022 & 0.26395 \\
15 & 1 & 1 & 0.930397 & 0.413252 \\
16 & 1 & 0.550914 & 0.372671 & 0.221643 \\
17 & 1 & 1 & 1 & 1 \\
18 & 0.952539 & 0.351603 & 0.400584 & 0.263737 \\
19 & 1 & 1 & 1 & 0.991215 \\
20 & 1 & 0.399693 & 0.403382 & 0.183825 \\
\hline
\end{tabular}

Table 4 Efficiencies of DMUs

In Table 5, the classifications and ranking of these DMUs are presented. 


\begin{tabular}{|c|c|c|c|c|c|l|c|}
\hline$D M U_{j}$ & $\bar{\theta}_{j}{ }^{1}$ & $\bar{\theta}_{j}{ }^{2}$ & $\bar{\theta}_{j}{ }^{3}$ & $\bar{\theta}_{j}{ }^{4}$ & $\bar{\theta}_{j}{ }^{3}$ & Classification & Rank \\
\hline 1 & 3.523761 & 1.846914 & 1.482492 & 1.259058 & 2.028056 & $\mathrm{E}++$ & 7 \\
2 & 1.020757 & 0.506559 & 0.455339 & 0.207883 & 0.547634 & $\mathrm{E}+$ & 14 \\
3 & 1.359532 & 0.971804 & 0.83911 & 0.52135 & 0.922949 & $\mathrm{E}+$ & 12 \\
4 & 5.224134 & 2.418946 & 2.518259 & 1.259423 & 2.85519 & $\mathrm{E}++$ & 5 \\
5 & 0.763021 & 0.666492 & 0.71051 & 0.618831 & 0.689714 & $\mathrm{E}-$ & 16 \\
6 & 1.245606 & 1.025448 & 1.059813 & 0.917738 & 1.062151 & $\mathrm{E}+$ & 11 \\
7 & 3.172361 & 1.463782 & 1.544707 & 0.728914 & 1.727441 & $\mathrm{E}+$ & 8 \\
8 & 4.812406 & 1.869024 & 2.825858 & 1.433282 & 2.735142 & $\mathrm{E}++$ & 6 \\
9 & 5.745185 & 2.703864 & 2.451013 & 1.238175 & 3.034559 & $\mathrm{E}++$ & 4 \\
10 & 19.90213 & 11.50958 & 15.19096 & 7.955503 & 13.63954 & $\mathrm{E}++$ & 2 \\
11 & 26.44258 & 20.38311 & 10.35878 & 8.209075 & 16.34839 & $\mathrm{E}++$ & 1 \\
12 & 0.496069 & 0.396102 & 0.403706 & 0.328345 & 0.406055 & $\mathrm{E}-$ & 20 \\
13 & 0.701987 & 0.53513 & 0.538834 & 0.449969 & 0.55648 & $\mathrm{E}-$ & 17 \\
14 & 0.724067 & 0.353113 & 0.368022 & 0.26395 & 0.427288 & $\mathrm{E}-$ & 19 \\
15 & 3.181636 & 1.168753 & 0.930397 & 0.413252 & 1.423509 & $\mathrm{E}+$ & 10 \\
16 & 1.297599 & 0.550288 & 0.372671 & 0.221643 & 0.61055 & $\mathrm{E}+$ & 13 \\
17 & 6.536229 & 2.65356 & 3.672324 & 2.158379 & 3.755123 & $\mathrm{E}++$ & 3 \\
18 & 0.952539 & 0.351603 & 0.400584 & 0.263676 & 0.492101 & $\mathrm{E}-$ & 18 \\
19 & 2.218415 & 1.349766 & 1.227325 & 0.991215 & 1.44668 & $\mathrm{E}+$ & 9 \\
20 & 0.977855 & 0.399693 & 0.403382 & 0.183825 & 0.491189 & $\mathrm{E}+$ & 15 \\
\hline
\end{tabular}

Table 5 Classification and Ranking of DMUs

Regarding Table 4and table 5, it can be seen that branches 1, 4, 8, 9, 10, 11and 17 are efficient in their worst condition there for they are placed in E++, and among these braches branch number 11 has the best rank, and branches 10, $17,9,4,8$ and 1 lie after it. It is also seen that branches $2,3,6,7,15,16$, 19 and 20 are put in $\mathrm{E}+$, because they are efficient in their best condition, but they are inefficient in their worst condition. Among the branches in E+, branch number 7 has the best rank, and after that lie branches 19, 15, 6, 3, 16, 2 and 20. And finally, since branches 5, 12, 13, 14 and 18 are inefficient in their best condition, they are placed in E-, and among them, branch number 5 has the best rank and branches 13, 18, 14 and 12 lie after it. Considering the fact that DMUs in $\mathrm{E}++$, and those in $\mathrm{E}+$ have a better rank that those in E-, the ranking of DMUs has been shown in table 5 .

\section{Conclusion}

Regarding table 5, we can observe that $D M U_{20} \in E^{+}$and $D M U_{5} \in E^{-}$ but $\theta_{5}>\theta_{20}$, and this is due to the fact that the data of $D M U_{5}$ are in a smaller interval than that of the data of $D M U_{20}$ and $e_{20}^{4}$ in much less than $e_{5}^{4}$ ; i.e., $e_{5}^{4}-e_{20}^{4}>e_{20}^{1}-e_{5}^{1}$.

Now we can rank the branches, according to the director,s opinion, in such a way that the higher the $\theta$ corresponding to a branch, the better its rank, regardless of which classes of $E^{++}, E^{+}$and $E^{-}$the given are placed in. It is 
obvious that if $D M U_{j} \in E^{++}$and $D M U_{i} \in E^{+}$or $D M U_{i} \in E^{-}$then $\theta_{j}>\theta_{i}$. According to what was mentioned, it can be observed that DMUs in $E^{++}$have better ranks than other DMUs. Thus, the branches in our example can be ranked as follows: $11,10,17,9,4,8,1,7,19,15,6,3,5,16,13,2,18,20,14$ and 12

\section{References}

P. Andersen, N.C. Petersen (1993), A procedure for ranking efficient units in data envelopment analysis. Mgmt. Sci. 39, 1261-1264.

R.D. Banker, A. Charnes, W.W. Cooper,(1984). "Some models for estimating technical and scale inefficiencies in DEA.", Mgmt. Sci. 30, 10781092.

A. Charnes, W.W. Cooper, E. Rhodes,(1978). "Measuring the efficiency of decision making units". European journal of operational research 2, 429-444.

W.W. Cooper, K.S. Park, G. Yu,(1999), "IDEA and AR-IDEA: models for dealing with imprecise data in DEA", Manage. Sci. 45, 597-607.

D.K. Despotis, Y.G. Smirlis,(2002)," Data envelopment analysis with imprecise data", European J. Oper. Res. 140 ,24-36.

G.R. Jahanshahloo, F. Hosseinzadeh Lotfi, M. Moradi,(2004),"Sensitivity and stability analysis in DEA with interval data", App. Math. Comput. $156,463-477$.

S.H. Kim, C.G. Park, K.S. Park,(1999)," An application of data envelopment analysis in telephone offices evaluation with partial data.", Computers \& Operations Research $26,59-72$.

Received: March 18, 2006 